\title{
Les synergies entre adaptation et atténuation en quelques mots
}

Bruno Locatelli

\section{Adaptation et atténuation}

\section{Que sont l'atténuation et l'adaptation ?}

L'atténuation et l'adaptation sont deux stratégies pour faire face au problème du changement climatique. L'atténuation est une intervention visant à réduire les sources ou augmenter les puits de gaz à effet de serre. L'adaptation est « un ajustement des systèmes naturels ou humains en réponse à des stimuli climatiques présents ou futurs ou à leurs effets, afin d'en atténuer les effets néfastes ou d'exploiter des opportunités bénéfiques » (IPCC 2001).

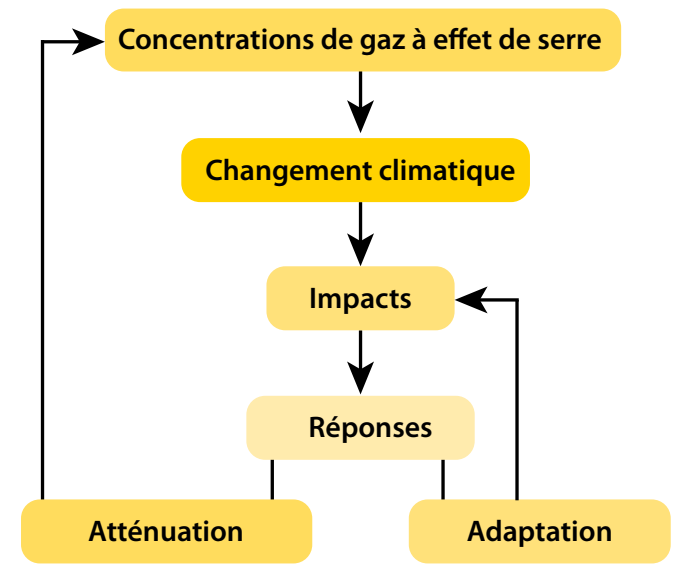

\section{Comment se différentient l'atténuation et l'adaptation?}

L'atténuation et l'adaptation présentent des différences notables, en particulier dans leurs objectifs. L'atténuation traite des causes du changement climatique (l'accumulation de gaz à effet de serre dans l'atmosphère), alors que l'adaptation concerne les impacts du changement climatique. Les deux approches sont nécessaires. D'une part, même si des efforts importants étaient faits sur l'atténuation, le climat continuerait à changer pendant les prochaines décennies, rendant nécessaire l'adaptation à ce changement. D'autre part, l'adaptation ne pourra pas éliminer tous les impacts négatifs et l'atténuation est cruciale pour limiter les changements dans le système climatique.

\section{Quelles sont les autres différences entre l'atténuation et l'adaptation?}

L'atténuation et l'adaptation concernent différentes échelles spatiales : même si le changement climatique est une préoccupation internationale, les bénéfices de l'adaptation sont locaux et ceux de l'atténuation sont globaux. L'atténuation et l'adaptation se différencient aussi par leurs échelles temporelles et les secteurs économiques concernés (Tol 2005).

\begin{tabular}{|c|c|c|}
\hline & Atténuation & Adaptation \\
\hline $\begin{array}{l}\text { Echelle } \\
\text { spatiale }\end{array}$ & $\begin{array}{l}\text { D'abord un problème } \\
\text { international, car } \\
\text { l'atténuation fournit } \\
\text { des bénéfices } \\
\text { globaux }\end{array}$ & $\begin{array}{l}\text { D'abord un } \\
\text { problème local, } \\
\text { car l'adaptation } \\
\text { fournit surtout des } \\
\text { bénéfices locaux }\end{array}$ \\
\hline $\begin{array}{l}\text { Echelle } \\
\text { temporelle }\end{array}$ & $\begin{array}{l}\text { A un effet à long } \\
\text { terme en raison de } \\
\text { l'inertie du système } \\
\text { climatique }\end{array}$ & $\begin{array}{l}\text { Peut avoir un effet } \\
\text { à court terme sur } \\
\text { la reduction de la } \\
\text { vulnérabilité }\end{array}$ \\
\hline \multirow[t]{2}{*}{ Secteurs } & $\begin{array}{l}\text { Une priorité pour les } \\
\text { secteurs de l'énergie, } \\
\text { des transports, de } \\
\text { l'industrie et des } \\
\text { déchets }\end{array}$ & $\begin{array}{l}\text { Une priorité pour } \\
\text { les secteurs de l'eau } \\
\text { ou de la santé et les } \\
\text { zones cỏtières }\end{array}$ \\
\hline & \multicolumn{2}{|c|}{$\begin{array}{l}\text { Atténuation et adaptation sont toutes } \\
\text { les deux pertinentes pour les secteurs de } \\
\text { l'agriculture et la forêt }\end{array}$} \\
\hline
\end{tabular}

\section{Références}

IPCC 2001 Climate change 2001. Synthesis report. Cambridge University Press.

Tol, R.S.J. 2005 Adaptation and mitigation: trade-offs in substance and methods. Environmental Science and Policy 8(6): 572-578.

\section{Forêts et atténuation}

\section{Quel est le rapport entre les forêts et l'atténuation ?}

Les forêts contribuent à l'atténuation grâce à leur capacité à absorber du carbone de l'atmosphère et à le stocker.
La déforestation et la dégradation forestière causent de 15 à $20 \%$ des émissions globales de gaz à effet de serre. Le rapport LULUCF (Utilisation des sols, changement 


\section{Forêts et atténuation : Exemples}
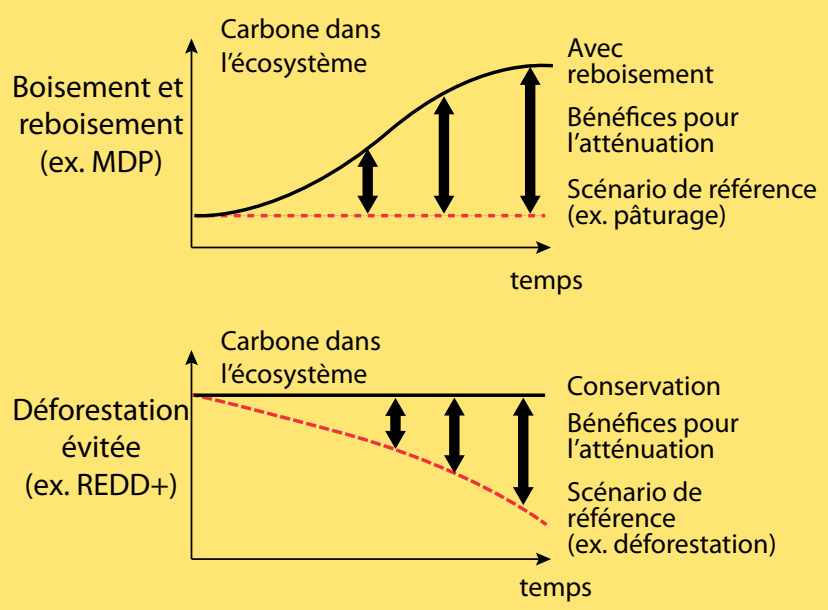

d'utilisation des sols et forêts) de I'IPCC distingue trois activités principales d'atténuation dans le secteur forestier le boisement (convertir en forêts des terres non forestières depuis une longue période), le reboisement (convertir en forêts des terres récemment déboisées) et la déforestation évitée (éviter la conversion de forêts en terres nonforestières) (Watson et al. 2000).

\section{Comment les mécanismes internationaux intègrent- ils les forêts pour l'atténuation?}

Seuls les projets de boisement et reboisement sont éligibles dans le cadre du Mécanisme de Développement Propre (MDP). La réduction des émissions provenant de la déforestation et la dégradation forestière (REDD), une initiative actuellement en bonne place dans l'agenda de négociations internationales, se base sur des incitations financières pour préserver les forêts et donc maintenir ou augmenter leurs stocks de carbone. Une approche REDD+ a été proposée pour financer non seulement la conservation de forêts mais aussi l'accroissement des stocks de carbone (par exemple par des boisements) et la gestion durable des forêts.

\section{Référence}

Watson, R.T., Noble, I.R., Bolin, B., Ravindranath, N.H., Verardo, D.J. et Dokken, D.J. 2000 IPCC special report on land-use, land-use change and forestry. Cambridge University Press, Cambridge, Royaume Uni.

\section{Forêts et adaptation}

\section{Quel est le rapport entre les forêts et l'adaptation ?}

Les liens entre les forêts et l'adaptation sont à double sens (Locatelli et al. 2010). Premièrement, comme le changement climatique va affecter les forêts, des mesures d'adaptation des forêts sont nécessaires pour réduire les impacts négatifs et maintenir les fonctions des écosystèmes (adaptation pour les forêts). Deuxièmement, les écosystèmes forestiers contribuent à l'adaptation en fournissant des services écosystémiques locaux qui réduisent la vulnérabilité des communautés locales et, plus largement, de la société, au changement climatique (forêts pour l'adaptation des populations).

\section{Qu'est ce que la vulnérabilité ?}

La vulnérabilité est un concept central à l'adaptation. La vulnérabilité au changement climatique est « le degré selon lequel un système est susceptible d'être affecté négativement par les effets du changement climatique (y compris la variabilité climatique et les extrêmes) ou est incapable d'y faire face » (IPCC 2001). La vulnérabilité dépend de l'exposition, de la sensibilité et de la capacité adaptative. Une forte exposition ou sensibilité et une faible capacité adaptative entrainent une forte vulnérabilité.

\section{Qu'est ce que l'adaptation basée sur les écosystèmes?}

Malgré son nom, l'adaptation basée sur les écosystèmes (ou EbA pour son acronyme en anglais, d'après 'ecosystembased adaptation) est une approche de l'adaptation centrée sur les humains. Elle a pour but de réduire la vulnérabilité

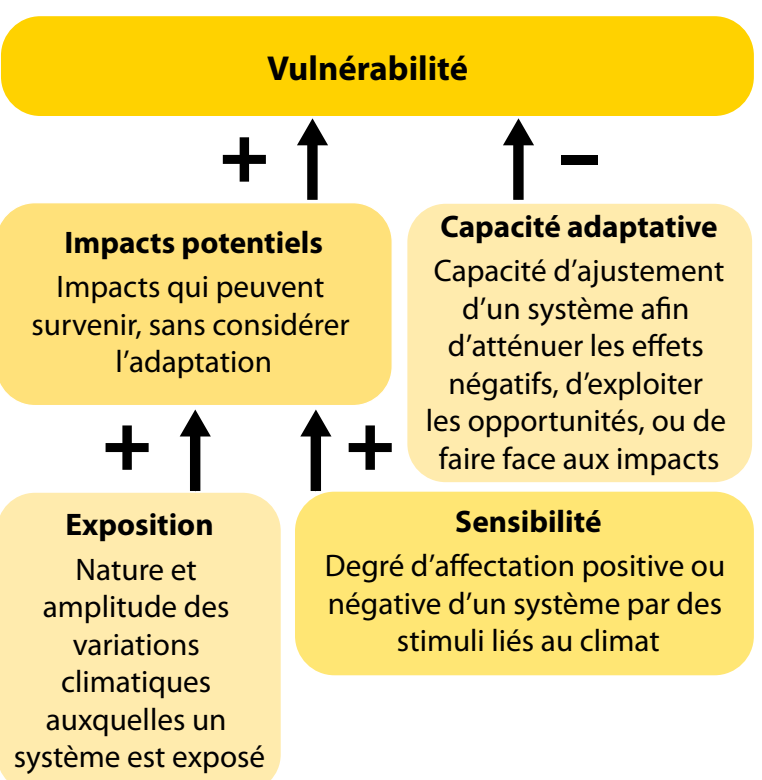

humaine à travers la fourniture de services écosystémiques. Il est de plus en plus reconnu que des écosystèmes bien gérés peuvent aider la société à s'adapter à la fois aux risques climatiques actuels et au changement climatique futur, en fournissant divers services écosystémiques, par exemple des mangroves protégeant les zones côtières contre les tempêtes ou les vagues, des forêts fournissant des produits utilisés comme filets de sécurité par les communautés quand l'agriculture est affectée par le climat, ou des forêts qui régulent la qualité de l'eau et les débits des rivières. Pour EbA, il est nécessaire de comprendre les vulnérabilités interdépendantes des humains et des écosystèmes et de considérer les écosystèmes dans un contexte large. 


\section{Adaptation basée sur les écosystèmes : Exemples}

Conservation du sol et hydro énergie en Amérique Centrale

- Augmentation de l'intensité des pluies et de l'érosion des sols.

- Augmentation de la sédimentation dans les barrages.

- La conservation des sols en amont fait partie de l'adaptation.

Forêts et communautés locales en Afrique Centrale

- Des évènements climatiques affectent les activités.

- Certains produits forestiers, moins sensibles que l'agriculture, représentent des filets de sécurité.

- La gestion forestière fait partie de l'adaptation.

Mangroves et zones côtières en Asie du Sud-est

- Vulnérabilité des côtes aux tempêtes, vagues et montée du niveau des mers.

- Mangroves : rôle protecteur et fourniture de biens.

- La gestion des mangroves fait partie de l'adaptation.

\section{Doit-on promouvoir les forêts pour l'adaptation des populations et en même temps mettre en place des mesures d'adaptation pour les forêts?}

Idéalement, oui. 'Promouvoir les forêts pour l'adaptation des populations' est pertinent dans la plupart des endroits, en particulier là où les populations dépendent directement des forêts pour leurs activités. Mais l'intégration de l'adaptation dans la gestion des forêts demande que ces forêts soient gérées ou conservées, ce qui n'est pas le cas partout. Pour s'assurer que les forêts fournissent les services écosystémiques pertinents pour la société (forêts pour l'adaptation), une gestion durable doit être d'abord assurée.
Ecosystèmes durables et résilients

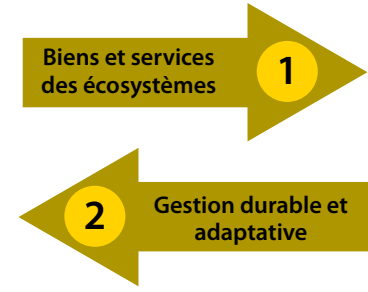

Société résiliente face au changement climatique et d'autres menaces
1 Forêts pour l'adaptation

2 Gestion durable pour une fourniture durable de services + Adaptation pour les forêts, si une gestion durable est en place

Quand les pressions immédiates sur les forêts (par exemple, la déforestation pour la conversion des terres) sont réduites, une perspective à plus long terme et le changement climatique peuvent être pris en compte (adaptation pour les forêts).

\section{Référence}

Locatelli, B., Brockhaus, M., Buck, A. et Thompson, I. 2010 Forests and adaptation to climate change: challenges and opportunities. En: Mery, G., et al. (éds) Forest and society: responding to global drivers of change. IUFRO World Series vol. 25: Vienne.

\section{Adaptation et atténuation : Les liens}

\section{Quels sont les liens entre l'adaptation des forêts et l'atténuation?}

Les projets forestiers d'atténuation (par exemple les projets REDD+) peuvent potentiellement faciliter l'adaptation des forêts au changement climatique, en réduisant les pressions anthropiques sur les forêts, en augmentant la connectivité entre forêts et en conservant des sites riches en biodiversité. Cependant, les gestionnaires de projets d'atténuation vont surement devoir incorporer de nouvelles mesures pour l'adaptation des forêts pour réduire les impacts du changement climatique sur ces forêts, car de tels impacts peuvent mettre en danger la permanence du stockage de carbone.

\section{Comment un projet d'atténuation peut-il influencer l'adaptation des populations ?}

Les projets forestiers d'atténuation peuvent avoir des impacts positifs sur les populations locales et leur capacité adaptative. Ils peuvent accroitre la fourniture de services écosystémiques aux communautés locales, diversifier leurs revenus et leurs activités économiques, développer des infrastructures ou des services sociaux, et renforcer les institutions locales (Caplow et al. 2010). Mais les impacts peuvent aussi être négatifs. Par exemple, des inquiétudes ont surgi quant à la possibilité que des projets REDD+ réduisent les droits et l'accès des populations locales à la terre et aux ressources forestières ou augmentent la dépendance des populations à des financements externes incertains.

\section{Comment un projet d'adaptation peut-il agir sur l'atténuation?}

Les projets d'adaptation peuvent affecter directement les écosystèmes et leurs stocks de carbone, donc agir sur l'atténuation. Les projets d'adaptation basée sur les écosystèmes peuvent avoir des bénéfices sur l'atténuation, en augmentant ou conservant les stocks de carbone. Les synergies entre services écosystémiques expliquent les impacts des projets d'adaptation sur l'atténuation. Par exemple, les mangroves contribuent à la fois à protéger les zones côtières et à stocker du carbone. Cependant, il peut y avoir des compromis entre le carbone et les services écosystémiques pertinents pour un projet d'adaptation. Par exemple, les priorités spatiales pour la conservation des services écosystémiques hydrologiques et pour le carbone peuvent différer. 
En plus des impacts directs des projets d'adaptation, d'autres impacts indirects peuvent être envisagés, si un projet d'adaptation évite le déplacement d'activités et la déforestation associée, par exemple si un projet d'adaptation agricole soutient la productivité agricole et réduit la conversion de forêt par l'expansion agricole.

\section{Référence}

Caplow, S., Jagger, P., Lawlor, K. et Sills, E. 2010 Evaluating land use and livelihood impacts of early forest carbon projects: lessons for learning about REDD+. Environmental Science and Policy 14: 152-167.

\section{Intégrer adaptation et atténuation}

\section{Quelles sont les principales raisons pour considérer à la fois l'adaptation et l'atténuation dans des projets forestiers ?}

L'atténuation a besoin de l'adaptation. Un projet REDD+ a plus de chances d'être durable et son carbone d'être permanent s'il met en œuvre des mesures d'adaptation pour les communautés locales et les écosystèmes. Intégrer l'adaptation peut aussi augmenter la légitimité locale du projet, car l'adaptation met l'accent sur les besoins locaux (Locatelli et al. 2011).

L'adaptation a besoin de l'atténuation. Un projet d'adaptation qui contribue à l'atténuation peut bénéficier de financements carbone et de renforcement de capacités par le biais d'instruments internationaux comme REDD+. Des bailleurs de fonds pour l'adaptation peuvent avoir une préférence pour des projets avec des bénéfices globaux, comme l'atténuation, en plus des bénéfices locaux pour l'adaptation.

\section{Qu'est ce qui peut faciliter l'intégration de l'adaptation et l'atténuation?}

Plusieurs facteurs peuvent faciliter l'intégration de l'adaptation et l'atténuation (Locatelli et al. 2011):

Les politiques nationales. Par exemple, les autorités nationales peuvent approuver les projets d'atténuation seulement s'ils intègrent l'adaptation.

Les politiques internationales. Jusqu'à maintenant, l'adaptation et l'atténuation ont été traitées séparément, même si certains pays ont demandé que "les mesures adaptation soient développées en considérant [...] les synergies entre adaptation and atténuation, et pour lesquelles les options REDD+ sont particulièrement pertinentes" (CNUCC 2009).
Des standards. Par exemple, les standards CCB (Climate Community Biodiversity), qui évaluent les impacts de projets d'atténuation, prennent en compte de façon explicite des critères d'adaptation (CCBA 2008).

La création de connaissances, la communication et le renforcement de capacités. Les acteurs de l'adaptation et l'atténuation forment des communautés séparées. II est nécessaire d'informer les acteurs de l'atténuation à propos de l'adaptation, et vice-versa, ainsi que de renforcer leurs capacités sur des méthodes et des outils appropriés. II y aussi un besoin pour plus de recherche : des méthodes, des outils et des données doivent être produits, par exemple sur le rôle des écosystèmes dans l'adaptation de la société ou sur les impacts de projets REDD+ sur les communautés locales et leur capacité adaptative.

\section{Références}

Locatelli, B., Evans, V., Wardell, A., Andrade, A. et Vignola, R. 2011 Forests and climate change in Latin America: linking adaptation and mitigation. Forests $2: 431-450$.

CNUCC 2009 Paper no. 27A. Nicaragua on behalf of Guatemala, Dominican Republic, Honduras, Panama and Nicaragua : adaptation. Proposal on the long-term agreement within the framework of the Bali Action Plan, in Ad hoc working group on long-term cooperative action under the convention, Sixth session, 1-12 June 2009. United Nations Framework Convention on Climate Change. Bonn, Allemagne.

CCBA 2008 The Climate, Community and Biodiversity Project Design Standards (CCB Standards), 2nd version. Conservation International.
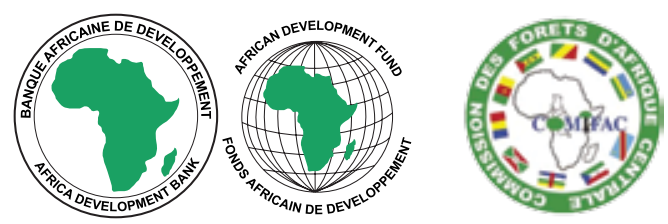

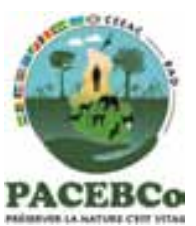

www.ForestsClimateChange.org 